\title{
LA INVESTIGACION ACTIVA COMO HERRAMIENTA PARA MEJORAR LA ENSEÑANZA DE LA QUÍMICA: NUEVOS ENFOQUES
}

\author{
DE JONG, 0 . \\ Universidad de Utrecht. CSME. Departamento de Enseñanza de la Química, Princetonplein 5. \\ 3584 CC Utrecht, The Netherlands.
}

\begin{abstract}
SUMMARY
In many European countries, chemistry education faces a number of important recurrent difficulties. For example, at the secondary school level, students' interest in chemistry is decreasing and teachers complain that repeated explanation and demonstration are not very effective, which frustrates them. At university level, lecture courses and laboratory courses are often disconnected and many student experiments are considered as boring «cookbook» problems. These and other recurrent difficulties have evoked the need to improve the quatity of chemistry education at both levels.

In this article, the contribution of educational research to an improvement of chemistry education is discussed. This research is considered as a tool for analysing teaching and learning problems, for elucidating backgrounds of these difficulties and for developing and evaluating new approaches in chemistry education.

Special attention is focused on two promising new research approaches. First, a new research instrument is presented: protocols of discussions during real classroom and laboratory sessions. Second, a new research design is presented: the developmental research approach. Both research approaches can contribute to the improvement of chemistry classroom/laboratory practices and chemistry teacher training.
\end{abstract}

\section{CRISIS DE LA ENSEÑANZA ACTUAL DE LA QUUIMICA}

\section{Problemas actuales}

En muchos países curopeos, la enseñanza de la química se encuentra frente a un cierto número de difictultades recurrentes importantes. Por lo que respecta a Holanda, país del que soy originario, muchas de las dificultades se pueden describir tal como las expongo a continuación.

En el nivel de la escuela secundaria, la química tiene una imagen negativa para muchos alumnos. Éstos consideran que es una disciplina tediosa y tienen dificultades para entender los conceptos y reglas fundamentales. Además, el interés de los alumnos por la química, en tanto que asignatura electiva para los exámenes finales, ha disminuido hasta un nivel bastante bajo (Tabla 1 ).
Algunas de las quejas perfectamente conocidas de los alumnos podrían expresarse en la siguiente forma: «Me sé de memoria esta fórmula química, pero no entiendo su significado». Muchos profesores se quejan de que las explicaciones y las demostraciones repetitivas no resultan muy eficaces y producen frustracjón.

En el nivel universitario, también está disminuyendo el número de estudiantes de química del primer curso (Tabla I). Los estudiantes se quejan de que en los cursos de laboratorio abundan los problemas aburridos propios de un «libro de cocina» en lugar de contener tareas interesantes que permitan explorar nuevas áreas de la 
química. Por otro lado, los educadores se quejan de que muchos estudiantes no son capaces de relacionar los cursos lectivos con los de laboratorio y de que, por ese motivo, no son capaces de aplicar sus conocimientos (téricos) a la química en el contexto del trabajo práctico.

Otra categoria de problemas se refiere a los planes de estudio de química. Las quejas más conocidas se refieren a la sobrecarga de temas, a su confusa estructura y a la falta de temas modernos. Además, la relación entre los planes de estudios de química del nivel secundario y del nivel superior es bastante tenue.

Una última categoría de problemas se refiere a la preparación de los profesores de química. El interés por convertirse en profesor de esta disciplina no es muy alto, al menos, no to suficiente como para satisfacer las necesidades inmediatas de nuevos profesores de química. Los cursos de iniciación para el profesorado no solucionan el alejamiento entre (cursos de) teoría y (clases de) prácticas. Y los cursos de reciclaje tienen dificultades para cambiar las concepciones y las estrategias didácticas actuales de los profesores de química.

En una palabra, los problemas educativos pueden encuadrarse en tres areas diferentes: cómo se enseña la química a los alumnos, diseño de planes de estudio y cómo se hace la formación inicial y el reciclaje de profesores. Los problemas que estamos considerando aparecen en eI contex to del sistema educativo holandés'. Sin embargo, estoy convencido de que estos problemas pueden detectarse también en España y en otros países (europeos). En mi opinión, hay una crisis que amenaza la actual enseñanza de la química.

Tabia I

Interés en química como asignatura electiva en Holanda.

\begin{tabular}{|cccc|}
\hline Año & \multicolumn{2}{c|}{$\begin{array}{c}\text { Nivel escolar secundario } \\
\text { Porcentaje de estudiantes } \\
\text { HAVO* }\end{array}$} & $\begin{array}{c}\text { Nivel universitario } \\
\text { Número de estudiantes } \\
\text { de primer curso }\end{array}$ \\
1983 & 39 & 47 & 903 \\
1988 & 31 & 40 & 1090 \\
1990 & 30 & 38 & 1220 \\
1992 & 28 & 37 & 1117 \\
1994 & 28 & 36 & 861 \\
\hline
\end{tabular}

* HAVO = Nivel general superior *VWO = Nivel preuniversitario

\section{Afrontar la crisis}

Para afrontar la presente crisis, creo que es necesario aplicar una estrategia de resolución de problemas que incluya los siguientes pasos:

- Describir y analizar en profundidad las dificultades para enseñar y aprender la química.

- Determinar, ampliamente, los posibles antecedentes de esas dificultades.
- Desarrollar y aplicar atentamente nuevas estrategias educativas, nuevos planes de estudio y cursos de formación inicial y de reciclaje.

- Evaluar sistemáticamente estos nuevos métodos educativos.

Respecto a cada una de estas actividades, la investigación en la enseñanza de la química puede desempeñar un papel muy importante. En este artículo, se discutirá la contribución de esta categoría de investigación a una mejora de la enseñanza de la química (y del profesorado). En la sección siguiente, se analizarán los actuales métodos de investigación y, en las secciones subsiguientes, se prestará especial atención a dos nuevos y prometedores métodos. Primeramente, se introduce un nuevo instrumento de investigación: los protocolos de discusión durante las clases y las sesiones de laboratorio. En segundo lugar, se presenta un nuevo esquema: el método de investigación evolutivo.

Ambos métodos pueden considerarse como partes metodológicas de la investigación activa, puesto que la exploración se centra en situaciones (educativas) reales y pretende contribuir al cambio de esas situaciones. En este artículo, solamente se elaborarán los métodos para el nivel de la escuela secundaria. Sin embargo, su valor para el nivel superior es también muy alto. Será el lector quien decida en qué casos es posible sustituir términos como «profesor (de secundaria)» y "clase" por los têrminos "catedrático o profesor (universitario)" y «laboratorio».

\section{MÉTODOS ACTUAIES DE INVESTIGACIÓN EN LA ENSENANZA DE LA QUIMICA}

\section{La línea de investigación en ciencia cognitiva}

Durante muchos años, e incluso actualmente, los entornos (teóricos) de la investigación en la enseñanza de la química se han visto fuertemente influidos por las teorías psicologicas generales acerca de la enseñanza y el aprendizaje. Hace algunas décadas, el paradigma fundamental era conocido como conductismo descriptivo, y englobaba teorías de estímulo-respuesta sobre la orientación de la conducta mediante el condicionamiento operativo (Skinner, 1953). Este punto de vista ha propiciado la aparición de estudios sobre cursos de química en los que está implicada la enseñanza programada: series de tareas con realimentación directa de las respuestas de estudiantes que aprenden de forma individual. Fin las dos últimas décadas, ha aparecido otro paradigma conocido como psicología cognitiva. Este método ha suscitado el interés hacia ciertos cursos que estaban basados, por ejemplo, en teorías del aprendizaje mediante descubrimientos dirigidos (Bruner, 1975) o basados en teorías de proceso de la información acerca del aprendizaje y de las condiciones de aprendizaje (Gagné, 1977).

Muchos estudios de ciencia cognitiva se centraron en los resultados del aprendizaje y, a menudo, utilizaron méto- 
dos cuantitativos para recoger y analizar los datos de la investigación. Con frecuencia, Ios instrumentos utilizados consistían en preguntas con diversas respuestas posibles y preguntas que exigían respuestas redactadas. De este modo, se pueden recoger con bastante facilidad grandes cantidades de datos y el análisis de las respuestas resulta una trabajo bastante sencillo, especialmente si se pueden aplicar métodos estadísticos. Por consiguiente, muchos estudios cuantitativos se hicieron a gran escala. Un vision general de la utilización de los métodos cuantitativos en la investigación educativa de la química puede verse en Nurrenbern y Robinson (1994).

En mi opinión, el valor del método de investigación en ciencia cognitiva, en cuanto a mejorar la química, queda restringido por el hecho de que las características que analizan muchos modelos educativo-psicológicos son muy generales para diseñar una enseñanza de la química bien fundamentada. Por ejemplo, es posible desarrollar varios cursos de química divergentes a partir de la misma teoría científica cognitiva. La inversa también es posible: un curso concreto de química puede basarse en varias teorías científicas cognitivas divergentes. Esta débil relación entre teoría (general) y práctica (concreta) puede explicarse de la siguiente manera: quienes desarollan cursos utilizan (a menudo de forma implícita) opiniones y conceptos básicos de química y de ta enseñanza de la química durante el proceso de diseño de nuevas estrategias educativas. Sus creencias y pensamientos acerca de aspectos educativos concretos de la química, a menudo, son más unas creencias personales que modelos generales de enseñanza y aprendizaje. Por consiguiente, es importante realizar estudios que tomen en consideración los aspectos relacionados con el contenido de la enseñanza de la química.

\section{La línea de investigación sobre áreas concretas}

Fn Ia última década, está aumentando el interés por el estudio de temas concretos de la enseñanza y aprendizaje de la química, tales como Ias reacciones ácido-base o los análisis volumétricos. La investigación sobre áreas concretas se está viendo fuertemente impulsada por el actual paradigma del constructivismo. Según este punto de vista acerca de la adquisición de conocimientos (Driver, 1989; Bodner, 1986), el aprendizaje es un proceso dinámico y social en el cual quienes aprenden construyen los significados, de forma activa, a partir de sus experiencias concretas, ligadas a su entendimiento previo y a su marco social. Se considera que el conocimiento y el aprendizaje tienen un Iugar fundamental. Es decir, el conocimiento es, en parte, un producto de la actividad, del contexto y de la cultura en los cuales se desarrolla y se utiliza.

Una implicación importante para la enseñanza es la idea de que los profesores de química deberían explorar las concepciones (previas) de los estudiantes acerca de los temas de química y deberían facilitar el aprendizaje mediante la creación de las condiciones que permitan el cambio conceptual (Posner et al., 1982). Además, los profesores de química deberían centrar su atención en los aspectos contextuales de la adquisición del conocimiento conceptual (Brown et al., 1989).

Muchos estudios sobre áreas concretas se han centrado en las concepciones que tienen los estudiantes acerca de los conceptos y reglas de la química (documentados por Pfundt y Duit, 1991). Estos estudios suelen contener métodos cualitativos para recoger y analizar los datos de las investigaciones. Este tipo de protocolos puede aplicarse invitando a los alumnos a expresar lo que piensan mientras realizan ciertas tareas (introspección). También se puede pedir a los alumnos, una vez terminada la tarea, que digan lo que pensaban mientras la realizaban (retrospección). En ambos casos, las afirmaciones de los alumnos pueden grabarse en cinta magnetofonica y transcribirse en forma de protocolo. El análisis de estos protocolos genera la posibilidad de explorar la comprensión de los estudiantes acerca de un concepto científico, más allá del conocimiento de la definición formal de ese concepto en particular.

Los estudios sobre áreas concretas se hacen, a menudo, a pequeña escala debido al tiempo que exigen los métodos de análisis. Bowen (1994) ofrece una visión general acerca de la utilización de los métodos de la entrevista y del protocolo "pensar en voz alta», en la investigación de la enseñanza de la química.

En mi opinión, la investigación sobre áreas concretas constituye una herramienta muy útil para mejorar la enseñanza de la química. Sin embargo, su valor depende de Ias características de los instrumentos de investigación que normalmente se utilicen. Las entrevistas y los protocolos «pensar en voz alta» pueden utilizarse antes o después de las sesiones de clase, pero no resultan muy adecuados para la investigación acerca de la enseñanzaaprendizaje de la química en su contexto habitual: el propio entorno de clase-laboratorio. Para este tipo de investigación, los llamados protocolos de clase son más efectivos. Estos protocolos pueden aplicarse registrando en cinta magnetofonica las discusiones de los alumnos y de los profesores en entornos educativos y transcribiéndo las intervenciones en forma de protocolo. Los protocolos así obtenidos pueden constituir una fecunda fuente de información, aunque su análisis no siempre resulte sencillo. También pueden utilizarse los protocolos para investigar acerca de las concepciones y actividades de los profesores.

Los estudios sobre áreas concretas que incluyen la utilización de los protocolos de clase son más bien escasos, si bien está creciendo el interés por ellos. En la siguiente sección, se presentan brevemente los resultados del análisis de protocolos en dos de esos estudios. Tales estudios se centran en la enseñanza y aprendizaje de temas de química de distinta naturaleza, en el rivel de la escuela secundaria. El primer trabajo trata del estudio de los cálculos estequiométricos (nivel de escuela secundaria: curso 10 ). El segundo estudio trata sobre la enseñanza de conceptos y reglas en electroquímica (nivel de escuela secundaria: curso 11). Ambos estudios forman parte de un programa de investigación del Centro de Enseñanza de las Cien- 
cias y de las Matemáticas (CSME) de la Universidad de Utrecht (Verdonk y Lijnse, 1993).

\section{ANÁLISIS DE LAS DIFICULTADES UTILIZANDO PROTOCOLOS DE CLASE}

\section{Estudio I: Cálculos estequiométricos}

En esta sección se presentan algunos resultados analíticos de los protocolos de enseñanza y aprendizaje acerca de los cálculos estequiométricos. Este análisis forma parte de un estudio presentado en otro lugar (De Jong, 1990, 1992); aquí solamente se describen los aspectos relevantes. El proyecto de investigación se llevó a cabo en el segundo año de química de la escuela secundaria (edad de los alumnos: 15-16). La estequiometría se había introducido el año anterior. Pero en este segundo año se introduce por vez primera a los estudiantes el concepto de mol.

\section{El concepto de mol}

Se piđió a los alumnos que discutieran el significađo de mol en tanto que número determinado de partículas concretas (significado microquímico) y de mol como masa específica de una sustancia (significado macroquímico). A continuación, los alumnos deberían utilizar el mol dentro de un contexto de estudio y análisis de reacciones químicas. Uno de los problemas utilizados a este propósito se presenta en la tabla II. Para hallar la solución a este problema, los alumnos tenían que realizar, por primera vez, una descripción estequiométrica propia, utilizando el concepto de $\mathrm{mol}$.

Los análisis de los protocolos de clase indican que los estudiantes desarrollaron un método particular de razonamiento. Este método puede ponerse de manifiesto analizando una discusión de clase representativa (Tabla II).

Durante el episodio registrado en clase, una de las estudiantes (Jacqueline) dice que el número de moles no va a cambiar (líneas 4,5 y 11 ). Al principio, hay otra estudiante (Marianne) que no está de acuerdo y propone una respuesta casi correcta (líneas 2 y 3 ), pero más adelante retira sus palabras (línea 12), utilizando eI argumento de la primera alumna (línea 11). Además, la primera (Jacqueline) no menciona el término mol (líneas 4 y 11). Sin embargo, Ia otra (Marianne) especifica ese concepto al principio (líneas 2 y 3 ) e, incluso, destaca su importancia mediante la analogía de una tarta y sus trozos (Íneas 6 y 7); al final, también se olvida mencionar el nombre de ciertas "entidades" relevantes (línea 12).

Los análisis de protocolos, como el que acabamos de ver, ponen de maniffesto un método particular de los alumnos para razonar acerca del mol, en el contexto de una reacción. Los alumnos utilizan una regla de conservación: la conservación del número de moles. Ello viene acompañado de la omisión de una especificación acerca
Tabla II

Una discusión de clase sobre el concepto de nol

Situación de clase

Se trata de resolver el siguiente problema:

"Al disolver las sales en agua, estas se separan en iones.

Al disolver 1 mol de cloruro de calcio se obtienen iones de calcio e iones de cloruro.

¿Cuántos moles de iones se obtienen en total. Explicalo."

Tres alumnos elaboran, individualmente, su respuesta y posturiormente las discuten de la siguiente forma.

\section{Protocolo}

OI Jacqueline: Tenemos justo un mol.

02 Marianne: Esperaun momento, yo tengo 2, porque hay I cakio;

$03 \quad$ aparece 1 mol de calcio y I mol de cloruro

04 Jacqueline: Ś́, pero, si pones I mol, no puede salir más

05 que 1 mol... incluso si está dividido.

06 Marianne: Si tienes una tarta y la cortas en 10 trozos, ya no sera

07 una tarta, ¿no? Serán to trozos, ¿,to?

08 Lien: Quizá te lo puedas comer.

09 Marianne: Sí, pero mira como es; es una sustancia,

pero se convierte en dos.

10 Lien: Sí, pero...

II Jacqueline: Pero, en ese caso la cantidad sigue siendo $1 \mathrm{~mol}$.. la cantidad total.

12 Marianne: Sí, pero... entonces se convierte... claro,

el total sigue siendo I mol

13 Lien: $\quad$ Sí, eso es lo que nos están pidiendo.

14 Marianne: Pero entonces aparece tal y tal un mol.

15 Iien: $\quad$ Sí.

16 Marianne: Iones de calcio y tal y tal un mol

17 Lien: Pero nos piden cuántos moles de iones aparecen en

18

19 Jacqueline: Sí.

20 Marianne: De acuerdo.

de las partículas. Los profesores involucrados en el estudio parecen no darse cuenta de la regla de los alumnos acerca de la conservación del número de moles.

En conclusión, en un contexto microquímico, el concepto de mol no tiene un significado claro para los alumnos; esta situación es ignorada por los profesores.

\section{Resolución de problemas estequiométricos}

También se pidió a los alumnos que resolvieran problemas estequiométricos típicos de libros de texto en los que intervinieran cantidades dadas y pedidas de reactivos y productos. Una parte fundamental de la solución de tales problemas es el planteamiento de una proporción estequiométrica adecuada. Esta proporción implica dos razones equivalentes entre cantidades de sustancias: una derivada de la ecuación de la reacción y la otra derivada de las sustancias dadas y pedidas.

El análisis de los protocolos de clase indican que la mayoría de los estudiantes utilizan un tipo de proporción estequiométrica formulada bien en términos de gramos o bien en términos de moles. Por ejemplo:

Se trataba de resolver los siguientes problemas: 
- ¿Cuántos gramos de oxígeno se necesitan para quemar completamente 40 gramos de carbono?

- Una solución de 3 litros de cloruro de calcio tiene una concentración de iones cloruro de $0,3 \mathrm{~mol} / \mathrm{litro}$. ¿Cuántos moles de cloruro de calcio se han utilizado para preparar la solución?

Respecto al primer problema, la mayor parte de los estudiantes utilizó una proporción estequiométrica en gramos; respecto al segundo problema, se utilizó con mayor frecuencia una proporción estequiométrica en moles.

El resultado fue que la elección, por parte de los alumnos, de un tipo de proporción en concreto variaba y dependía del modo en que se expresaba la cantidad de sustancia dada (gramos o moles).

No obstante, para los profesores involucrados en el estudio, resultaba bastante normal preferir y prescribir sólo un tipo determinado de proporción: en moles. Su razonamiento era que la utilización de un algoritmo estándar, siempre el mismo, puede evitar confusiones a los estudiantes.

En conclusión, la preferencia de los profesores no se correspondía demasiado bien con la preferencia de los estudiantes.

\section{Estudio II: células electroquímicas}

En esta sección presentamos algunos resultados del análisis de protocolos sobre la enseñanza y el aprendizaje de las células electroquímicas. Este análisis forma parte de un estudio presentado en otro lugar (Acampo y De Jong, 1994); aquí solamente se describen los aspectos relevantes. El proyecto de investigación se llevó a efecto en el tercer año de química de la escuela secundaria (edad de los alumnos: 16-17). La electroquímica se había introducido en años anteriores, especialmente las reacciones de oxidación-reducción. Pero en este tercer año se introducen por vez primera a los al umnos conceptos relacionados con las células electroquímicas.

\section{Signo de la carga de un electrodo (media célula)}

Los profesores involucrados en el estudio introdujeron en clase el concepto de carga de un electrodo (semipila). Se điscutió la de Daniel (célula galvánica de zinc-cobre) y se utilizaron expresiones como uel zinc es negativo», así como «el cobre es positivo». De este modo, pretendían utilizar el nombre de ciertas sustancias para designar ciertos electrodos. No obstante, esta forma abreviada de formulación parecía suscitar difícultades entre los estudiantes en cuanto a su comprensión de la cinética đe las partículas en el entorno de un electrodo. Por ejemplo:

Un profesor explica una célula galvánica de zinc-cobre y utiliza la expresión «el cobre es positivo». Además, explica, mediante la siguiente media reacción, la constatación de que el electrodo de cobre se ha hecho más pesado: $\mathrm{Cu}^{2+}(\mathrm{aq})+2 \mathrm{e}-->\mathrm{Cu}(\mathrm{s})$.
Según los estudiantes, esta reacción implica que los iones positivos de cobre tenían que ganar electrones al desplazarse hacia un electrodo calificado de positivo. Para un gran número de estudiantes, este modelo cinético suscita un gran conflicto cognitivo. Dicho modelo es contrario a las leyes eléctricas de la repulsión entre cuerpos con carga del mismo signo.

Los profesores estaban utilizando una terminología que resultaba demasiado abreviada. En el caso de celdas galvánicas, una expresión como «el cobre es positivo» se refiere implícitamente al signo de la carga del electrodo de cobre respecto al electrodo de zinc. La estrategia de los profesores de utilizar formas abreviadas de formulación parecía suscitar gran confusión entre los alumnos.

\section{La regla para predecir el signo de la carga}

Los profesores hablaban también de la relación entre el tipo de la semireacción y el signo de la carga de un electrodo. Introducían esta relación mostrando una pila de Daniel (celda galvánica). Los profesores explicaban que una diferencia en la fuerza de los reactivos en cuanto a la capacidad de ceder electrones determina el tipo de semireacción en cađa electrodo y, por consiguiente, el signo de la carga del electrodo. Después de este ejemplo introductorio, explicaban a los alumnos que la relación discutida tenía un valor más general. A continuación, pedían a los alumnos que predijeran los signos de las cargas del electrodo de algunas otras celdas. Como herramienta, los alumnos solamente podían utilizar una tabla que contuviera datos electroquímicos ${ }^{2}$.

Sin embargo, los alumnos tuvieron muchas dificultades para predecir los signos de las cargas de los electrodos. Especialmente en el caso de potenciales de electrodos estándar đel mismo signo. Por ejemplo:

Para trazar un diagrama de potencial de una celda galvánica de zinc-hierro, el profesor selecciona los datos significativos de la tabla adecuada de potenciales electroquímicos. En la parte baja del điagrama, escribe: ' $\mathrm{Zn} /$ $\mathrm{Zn}^{2+} ; \mathrm{V}^{\circ}=-0,76 \mathrm{~V}$; electrodo menos. En la parte alta, escribe: $\mathrm{Fe} / \mathrm{Fe}^{2+} ; \mathrm{V}^{\circ}=-0,44 \mathrm{~V}$; electrodo más.

Para los estudiantes, resultaba difícil entender que una semireacción que incluya un potencial de electrodo estándar negativo pueda producir una carga positiva en un electrodo.

En conclusión, la estrategia que seguían profesores, de generalizar a la categoría de regla una relación particular, suscitaba muchas dificultades a los estudiantes y puede considerarse como prematura.

\section{La regla para predecir reacciones de electrodos}

También había inconvenientes respecto a la relación mencionada anteriormente en el momento de enseñar las célutas electrolíticas. Los profesores postulaban la regla de que el agente oxidante más fuerte gana electrones en 
el electrodo negativo (y el agente reductor más fuerte pierde electrones en el electrodo positivo). Los profesores introducían esta regla, fundamentalmente química, mediante un sólo experimento.

La regla establecida suscitaba mucha confusión entre los estudiantes. Consideraban más atractiva una regla fundamentalmente física. Esta regla rival implica la idea de que las partículas cargadas son atraídas por los electrodos de carga opuesta. En el caso de un electrodo negativo, esta regla implica que cualquier ion positivo es mejor reactivo que un agente neutro, como, por ejemplo, el $\mathrm{H}_{2} \mathrm{O}$. El episodio de clase expuesto en la tabla III refleja una discusión típica acerca de la regla en cuestión.

Fn el episodio registrado, el alumno prefiere utilizar una regla física de atracción entre entes con carga opuesta (línea 4). Sin embargo, el profesor razona a partir de un contexto químico y utiliza una regla distinta (líneas 3 y 5$)$.

En conclusión, el método de los profesores consistente en razonar a partir de un contexto cuyo significado resulta distinto del contexto de los alumnos no es de gran ayuda para estos últimos en cuanto a su comprensión de los conceptos y reglas electromecánicos.

Tabla III

Una discusión de clase sobre predicción de reacciones con electrodos.

\section{Situación de clase \\ Tras observar la electrólisis de una solución de $\mathrm{KBr}$ entre electrodos de carbono, los alumnos deben describir las semireacciones. \\ Protocolo \\ 01 Profesor: \\ 02 Estudiante: \\ En este caso, ¿cuál es el mejor agente oxidante? \\ 03 Profesor: \\ $\mathrm{mm}, \mathrm{mm} 2 \mathrm{H} O$ \\ El agua es el mejor agentc oxidante [...] \\ El electrodo menos, el agua produce... $\mathrm{H}_{\text {,... }}$ por tanto, el gas que habéis visto es hidrogeno... \\ (04 Estudiante: Pero el potasio más es atraído y el agua no... \\ os Profesor: [...] Basta con tomar agua, cosa bastante común según las reglas.}

\section{ANTECEDENTES DE LAS DIFICULTADES EN LA ENSENANZA Y APRENDIZAJE DE LA QUIMICA}

\section{Paradigma experto-principiante}

Los resultados de estudios sobre áreas concretas, tales como los estudios de la sección anterior, han puesto de relieve muchas dificuItades para Ia comprensión mutua entre profesores y alumnos. Tales dificultades pueden explicarse utilizando el modelo clásico del «triángulo didáctico». De acuerdo con este modelo, una situación de clase puede considerarse como una parrilta de rela- ciones entre profesor, alumno y tema de la asignatura (por ejemplo, conceptos, reglas o procedimientos de química). Este modelo puede perfilarse más mediante la siguiente suposición: en el caso de una discusión entre un profesor y un alumno, puede parecer que han alcanzado un acuerdo acerca de algún tema, mientras que, de hecho, cada uno de ellos está aplicando una interpretación basada en un punto de vista diferente.

Por ejemplo, si un profesor y un alumno están hablando sobre átomos, el profesor puede estar pensando en partículas que tienen las características de un modelo científico, mientras que el alumno puede estar pensando en las partículas más pequeñas de una sustancia que conservan todas las características, como el color, el olor, ctc. Fn ese caso, el profesor está razonando desde un punto de vista más formal, mientras que el alumno lo hace desde un punto de vista más concreto. Tales diferencias de puntos de vista pueden constituir un obstáculo importante en el proceso de mutuo entendimiento.

Las diferencias de punto de vista pueden explicarse a partir de diferencias de posición respecto al tema de la materia que se esté considerando. Un ejemplo podrá ilustrar esta cuestión. Según uno de los episodios de clase acerca de la electroquímica (seccion 3.2), el profesor utiliza la expresión «el cobre es positivo", refiriéndose implícitamente al signo de la carga del electrodo de cobre respecto a otro electrodo. La expresion del profesor puede considerarse como una forma de hablar abreviada, bastante común entre expertos. Sin embargo, esta forma de hablar no parece ayudar mucho a los alumnos a entender nuevos conceptos.

\section{Contenido y estructura de los temas escolares de química}

Los resultados de estudios sobre áteas concretas han puesto también de relieve que aparecen muchas dificultades de aprendizaje debido a las características específicas de los temas de química. En muchos casos, se ha concluido que el contenido de estos temas no encujaba demasiado bien dentro de los umarcos alternativos" de los estudiantes. Por ejemplo, en el caso del tema de los cálculos estequiométricos, resultaba que los alumnos razonaban a partir de un punto de vista básico de «conservación», en este caso de la conservación del número de moles (Tabla II). La respuesta que daban ( $1 \mathrm{~mol}$ de cloruro de calcio da, en total, I mol de iones) es erronea desde un punto de vista de experto. En cambio, esa respuesta es correcta cuando no se consideran las partículas relevantes. Ésa es la razón por la que los alumnos no percibían suficientemente la necesidad de especificar las partículas. Para ellos, bastaba con utilizar su principio general básico de conservación al que estaban acostumbrados. Sin embargo, este marco no se adecua al significado de mol como número concreto de partículas que deben especificarse. Para los alumnos, está característica del mol tenía escasa significación.

También se puso de manifiesto que la estructura de los temas escolares de química suscita, de hecho, grandes 
dificultades educativas. Esta estructura es, fundamentalmente, el resultado de los conceptos y experiencias de los expertos (por ejemplo, de los profesores de química), acerca de la química. No obstante, parece que la estructura «experta» de muchos temas no encaja suficientemente en una estructura significativa de las condiciones de aprendizaje. Este hecho puede ilustrarse utilizando la descripción de un modelo de condiciones de aprendizaje desarrollado por Posner y otros (1982). Este modelo contiene cuatro condiciones para aprender nuevos conceptos y reglas. Según la primera condición, es importante que los estudiantes tengan ocasión de empezar a partir de sentimientos de insatisfacción respecto a los conceptos que ya tienen. Deberían notar que sus conocimientos no son suficientemente adecuados para describir, explicar o predecir un fenómeno nuevo. Según las otras tres condiciones de aprendizaje, es importante que un nuevo concepto o regla pueda convertirse en inteligible, aceptable y provechoso para el alumno. La falta de una, o más, de estas cuatro condiciones puede dificultar las actividades de aprendizaje.

En el caso de la enseñanza de la química, resulta que el cumplimiento de todas las condiciones viene entorpecido por la estructura experta de los temas. De forma especial, existe una falta de interés por cumplir la primera condición para el aprendizaje. Por ejemplo, en el caso del tema de las células electroquímicas (sección 3.2), el episodio de clase indica que el alumno no siente la necesidad de aceptarle al profesor una nueva regla (química) cuando éste dice «Basta con tomar agua, cosa bastante común según las reglas». EI alumno prefiere utilizar una regla (física) que ya conoce, que le resulta más inteligible y aceptable, y dice: «Pero el potasio más es atraído y el agua no». Además, en el caso del planteamiento de una proporción estequiométrica apropiada (sección 3.1), los alumnos no ven la necesidad de utilizar siempre el mismo tipo de proporción.

En resumen, las dificultades en la enseñanza y aprendizaje de la quimica pueden ponerse de relieve observando cómo razonan los estudiantes mientras aprenden química. Se comprueba que el contenido de muchos temas no concuerda demasiado bien con las concepciones (previas) de los estudiantes y que la estructura de los temas no facilita el desarrollo de las adecuadas condiciones de aprendizaje.

\section{PERSPECTIVAS PARA LA MEJORA DE LA ENSENANZA DE LA QUIMICA}

\section{Unión íntima entre investigación y desarrollo}

No hay duda de que es necesario promover un análisis crítico del contenido y estructura de los temas actuales en las clases de química. Los conocimientos expertos (del profesor) sobre la química no son una base suficiente para la reconstrucción de los temas a enseñar. Tal reconstrucción niega la importancia y el papel del cono- cimiento del (alumno) principiante en el proceso de cambio conceptual. Las cuestiones clave son cómo reestructurar los temas escolares de química, cómo guiar el aprendizaje del alumno y cómo alcanzar una unión fecunda de la enseñanza y el aprendizaje de una asignatura concreta, desde una perspectiva constructivista. El diseño de ese tipo de enseñanza y aprendizaje debería ser un proceso empirico de investigación y desarrollo íntimamente relacionados. Un método nuevo y prometedor en esta área es el conocido como «método de investigación evolutiva» (Verdonk y Lijnse, 1993; I.ijnse, 1995).

Con este método, el desarrollo de planes de estudio a pequeña escala se relaciona con la investigación en profundidad de los procesos de enseñanza-aprendizaje que tienen lugar en el auta, tanto en relación al contenido como al contexto. La estructura de las actividades de investigación puede describirse como ciclos reiterados en espiral (en la tabla YV, se presenta un modelo del método cíclico). El punto de partida de este tipo de investigaciones es la evaluación de las situaciones educativas existentes. Se formulan preguntas exploratorias junto con una reflexión acerca de la química y de la ensefianza de la química. A continuación, se desarrollan y se aplican nuevas estrategias educativas y nuevos materiales. Durante las clases lectivas y las sesiones de Iaboratorio, se indaga acerca de los procesos educativos y de aprendizaje. Las características de Ia investigación evolutiva son fundamentalmente cualitativas. Cintas de audio y de vídeo constituyen importantes instrumentos de investigación para obtener protocolos de las discusiones de clase o de Iaboratorio.

Durante los últimos años, se ha llevado a cabo un cierto número de estudios de este tipo en Holanda acerca de la enseñanza de la química. En la sección 3, se ha presentado parte de dos de estos trabajos. Ambos estudios han generado lo que se llama nuevas estructuras didácticas de Ios temas de química. La estructura didáctica de los «cálculos estequiométricos» implica un método, mediante planteamiento de problemas, en el que se justifica la introducción del concepto de mol y se establece una estrecha conexión entre los contextos de las sustancias, de las partículas y de las reacciones para hacer que la utilización del mol sea inteligible, aceptable y provechosa para los alumnos (De Jong, 1990). La estructura didáctica de las «células electroquímicas» implica un método, mediante planteamiento de problemas, en el que la atención se centra, más de lo que es habitual, en modelos descriptivos de los cambios que ocurren en una céluta, y se presta menos atención a los cálculos, incluyendo Ia utilización de la ecuación de Nernst (Acampo y De Jong, 1994). Como punto de arranque, se utilizan algunos experimentos, mediante planteamiento de problemas, acerca de células simples, tal como los propuestos por Barral, Fernández y Otero (1992). Otros ejemplos de estudios evolutivos, a nivel secundario, tratan los conceptos de reacción química (De Vos y Verdonk, 1987) y de equilibrio químico (Van Driel, 1990).

Un ejemplo reciente de estudio evolutivo, de nivel universitario, trata ciertos métodos de síntesis de sustancias orgánicas (Van Keulen, 1995). La estructura didáctica 
implica la sustitución de los experimentos de «ibro de cocina» por experimentos que implican planteamiento de problemas. Los nuevos experimentos se centran en la simulación đe situaciones de experimentación química. Se propone a los estudiantes de primer curso que formtlen sus propias hipótesis y que diseñen y lleven a cabo pequeños experimentos para comprobar sus hipótesis. En ese curso, no se insta a los estudiantes para que aprendan un cierto número de síntesis, sino a que aprendan a realizar síntesis.

Fn mi opinión, la investigación evolutiva constituye un método prometedor para mejorar la enseñanza de la química. Los resultados de la investigación pueden contribuir a desarrollar modelos didácticos bien estructurados, actividades de clase bien fundamentadas y cursos provechosos para los profesores de química. Además, si se Ileva a cabo la investigación evolutiva en el nivel secundario, tanto como en el nivel universitario, los resultados pueden también contribuir a reforzar la conexión entre ambos niveles de planes de estudio de química.

Tabla IV

El método cíclico.

\begin{tabular}{|l|} 
* Evaluación de las actuales \\
situaciones educativas \\
la enseñanza y el aprendizaje de la ciencia
\end{tabular}

\section{Cambio de las concepciones $y$ acciones de los profesores}

En la (mejora de la) enseñanza de la química, los profesores desempeñan un papel central. Pueden ser considerados como expertos en química (escolar), a pesar de que su conocimiento acerca de algunos temas, tales como los modelos cognitivos de la ciencia (Izquierdo, 1995), podrían mejorar. Además, en ocasiones, la condición de expertos puede ser causa, involuntaria, de numerosos impedimentos para las actividades educativas (véase sección 3 ).

Los resultados de los estudios evolutivos han puesto también de manifiesto que muchos profesores de quími- ca tienen sentimientos y actitudes ambivalentes respecto a sus estrategias educativas habituales (De Jong, 1992; De Jong y Acampo, 1994). Por ejemplo, por un lado, creen que es importante imponer a Ios estudiantes el conocimiento de conceptos y reglas con el fin de evitar pérdidas de tiempo y que los alumnos distraigan la atención, especialmente en el caso de trabajar con clases numerosas. Pero, por otro lado, creen también que es importante para los alumnos aprender de forma activa y desarrolfar nuevos conocimientos observando $a$ interpretando por sí mismos los fenómenos. Los profesores son también ambivalentes respecto a su costumbre de usar una terminología drásticamente abreviada. Creen que es importante utilizar expresiones precisas para designar conceptos y reglas, y, por otro lado, consideran que resulta muy atractivo (y habitual entre expertos) utilizar expresiones abreviadas.

Para una correcta actividad educativa, los profesores necesitan tres categorías de conocimientos: conocimiento del tema de la materia, conocimientos generales de pedagogía y conocimiento pedagógico de los contenidos (Shulman, 1987). En mi opinión, los profesures de guímica necesitan más conocimientos pedagógicos de los contenidos; es decir, más conocimiento concreto de los contenidos y del contex to sobre la forma de presentar y formular los temas de química con el fin de hacer que esos temas resulten comprensibles para los estudiantes. Una buena formación inicial y el reciclaje en activo de los profesores podría ser de gran ayuda. Sin embargo, muchos de los cursos de formación y reciclaje centran gran parte de su atención en estrategias educativas más generales. Estas estrategias no se basan en un análisis profundo de contenidos y contextos concretos de los procesos de enseñanza-aprendizaje. Por tanto, la mayoría de los cursos no enlazan adecuadamente con las necesidades (prácticas) de los profesores. Existe un vacío entre teoría (cursos) y práctica (en las aulas).

Para mejorar la educación de los profesores de química, los cursos deberían estimular a los profesores a ampliar y modificar sus conocimientos pedagógicos de los contenidos relativos a los conceptos y reglas concretos a enseñar. Podrían recomendarse las siguientes líneas orientativas básicas:

a) Los profesores de química deberían saber mucho mas acerca de las concepciones y actividades (previas) de los estudiantes.

Se debería animar a los profesores a que leyeran y discutieran estudios sobre las concepciones y actividades (previas) de los estudiantes. También se les debería invitar a observar las actividades de aprendizaje de los estudiantes y a escuchar, tanto como sea posible y con una mentalidad abierta, las intervenciones de los estudiantes. Escuchar y observar a los estudiantes puede resultar más fácil si se les agrupa en pequeños grupos de trabajo.

b) Los profesores de química deberían aplicar estrategias educativas más precisas en cuanto a contenido y contexto. 
Se debería invitar a los profesores a dedicar mucha más atención a los significados de cada concepto o regla en un contex to concreto. Se les debería animar a desarrollar estrategias para la enseñanza de esos conceptos y reglas desde una perspectiva constructivista. Para ello, se puede pedir a los profesores que ofrezcan a los estudiantes un cierto número de experimentos orientados hacia la investigación y otra serie de tareas que impliquen el planteamiento de problemas. Los profesores podrían organizar discusiones en pequeños grupos, seguidas de una sesión plenaria final.

c) Los profesores de quimica deberían sermás conscientes de sus propias convicciones educativas y de sus actuaciones en clase.

Se debería animar a los profesores a que reflejaran sus propias concepciones y sus propias maneras de actuar. Se les puede pedir que consideren dos visiones opuestas en cuanto al papel del profesor: como directo transmisor de conocimientos o como estimulador de un proceso de desarrollo del conocimiento. Sus reflexiones pueden resultar más practicables si se escriben sobre papel algunas experiencias y algunos problemas importantes relativos a las propias clases y a notas tomadas durante discusiones con otros colegas.

Con el fin de ayudar a los profesores a llevar a caboestas recomendaciones, es importante ofrecerles cursos adecuadamente diseñados sobre la enseñanza de temas de química. Estos cursos deberían excluir métodos de formación «dirigista» para permitir una participación activa de los profesores. Ello quiere decir que los cursos deberían tener las características de la llamada formación interactiva de profesores (De Jong, 1992). No solamente es importante desarrollar cursos para profesores de química tal como los descritos, sino también investigar acerca del impacto de tales cursos sobre los profesores. Concretamente, se podría centrar la atención en los procesos de aprendizaje de los profesores en cuanto al cambio de sus concepciones educativas y en cuanto a la reorientación de las actividades pedagógicas. Los resultados de esta investigación podrían contribuir a la mejora de la enseñanza de la química.

\section{COMENTARIOS FINALES}

El profesor de química es uno de los «actores» más importantes en el proceso de mejora de la enseñanza de la química. En este proceso, puede desempeñar diversos papeles. Puede ser un consumidor de los resultados de la investigación. También puede ser productor de nuevos materiales y estrategias educativas, por ejemplo, participando en proyectos de investigación evolutiva. El profesor puede actuar también como escritor de libros de texto o como consultor. Uno de los papeles más importantes es el de profesor en tanto que investigador. Ello implica que el profesor investigue sobre la enseñanza y el aprendizaje en sus propias clases. Esta actividad investigadora a microescala puede llevarse a cabo según los pasos del método cíclico (sección 5.1). El profesor puede utilizar los resultados de su propia indagación con el fín de ser consciente de las concepciones de sus alumnos, así como de sus propias concepciones, y de este modo modificar las actividades dentro del aula. En conclusión, los estudios a microescala son también herramientas importantes para la mejora de la enseñanza de la química.

En el nivel secundario, los profesores holandeses de química deben seguir un curso de formación del profesorado en química para llegar a ser profesores cualificados. En la enseñanza superior, no es habitual que un profesor universitario siga un curso de estas características. La mayor parte de su tiempo se dedica a la investigación. En mi opinión, una mejora de la enseñanza universitaria de la química exige la promoción de cursos que se centren en un más profundo desarrollo de los conocimientos de contenido de la enseñanza de los profesores universitarios. Este año, la Universidad de Utrecht ha introducido una medida de cualificación educativa básica que implica que todos los profesores universitarios deben seguir un breve curso sobre la enseñanza de su asignatura. Los profesores asistentes tienen que preparar un dossier que contenga una descripciôn de sus experiencias educativas. Estos dossiers se someten a evaluación. La iniciativa de Utrecht puede resultar interesante para otros países que deseen mejorar la enseñanza superior.

Por último, un comentario sobre la mejora de la investigación sobre la enseñanza de la química. En muchos países (europeos), casi todos los grupos importantes de investigación son pequeños. La colaboración entre esos grupos está creciendo. En mi opinión, para mejorar la investigación sobre la enseñanza de la química, esa colaboración internacional no es solamente necesaria, sino recomendable y provechosa. Queda mucho trabajo por hacer.

\section{AGRADECIMIENTOS}

El autor agradece la ayuda y sugerencias recibidas por parte del editor y de los censores anónimos de Enseñanza de las Ciencias.

\section{NOTAS}

1 En el actual sistema educativo holandés, no se enseña química en la enseñanza primaria.

2 Esta tabla contiene una lista de medias ecuaciones emparejadas acompañadas de potenciales de electrodos estándar. I a tabla se ha utilizado anteriormente para deducir la fuerza relativa de los agentes oxidantes y reductores (en el caso de la predicción de reacciones redox directas).

* Este artículo ha sido traducido del inglés por J. Tortella. 


\section{REFERENCIAS BIBLIOGRÁFICAS}

ACAMPO, J. J. C. y DE JONG, O. (1994). Chemistry teachers» learning processes: a study of teacher training and reflection on classroom activities, en Schmidt, H.J. (ed.), Problem Solving and Misconceptions in Chemistry and Physics, pp. 229-238. Hong Kong: ICASE.

BARRAL, F.L., FERNÁNDEZ, E.G.R. Y OTERO, I.R.G. (1992). Secondary students' interpretations of the process occurring in an electrochemical cell. Journal of Chemical Education, 69, pp, 655-657.

BODNER, G.M. (1986). Constructivism: a theory of knowledge. Journal of Chemical Education, 63, pp. 873-878.

BOWEN, C.W. (1994). Think-aloud methods in chemistry cducation. Journal of Chemical Education, 71, pp. 184-190.

BROWN, J.S., COLLINS, A. y DUGUID, P. (1989). Situated cognition and the culture of learning. Educational Researcher, 18, pp. 32-42.

BRUNER, J.S. (1975). Towarda Theory of Instruction. Cambridge Mass.: Harvard University Press.

DE JONG, O. (1990). Towards a more effective methodology or research on teaching and learning «chemical calculations", en Schmidt, H.J. (ed.), Empirical Research in Mathematics and Science Education, pp. 106-121. Hong-Kong: ICASE.

DE JONG, O. (1992). Interactive teacher training; implications for an in-service course in teaching problem solving, en Vonk, J.H.C. et al. (eds.). New Prospects for Teacher Education in Europe Il, pp. 131-139. Amsterdam: VU Press.

DE JONG, O. y ACAMPO, I.J.C. (1994). Teaching bridge concepts in changing science contexts: a case study of teachers» strategies and conceptions, en Voorbach, J.T. (ed.), Teacher Education, 10, pp. 40-48. Academisch Boeken Centrum, De Lier.

DE VOS, W, y VERDONK, A.H. (1987). A new road to reactions, part 5. Joumal of Chemical Education, 64, pp. 1010-1013.

DRIVER, R. (1989). Changing conceptions, en Adey, P. (ed.), Adolescent Development and School Science, pp. 79-99. Londres: Falmer Press.
GAGNÉ, R.M. (1977). The Conditions of Learning. Nucva York: Holt, Rinchart \& Winston.

IZQUIERDO, M. (1995). Cognitive models of science and the teaching of science: history of science and curriculum, en Psillos, D. (ed.), Proceedings of the Second European Ph. D. Summerschool. Tesalónica: University of Thessaloniki (En prensa.)

LIJNSE, P. (1995). «Developmental rescarch» as a way to an empirical based adidactical structure» of science. Science Education, 79, pp. 189-199.

NURRENBERN, S.C. y ROBINSON, W.R. (1994), Quantitative research in chemical education, en Journal of Chemical Education, 71, pp. 181-183.

PFUNDT, H. y DUIT, R. (1991). Students' alternative frameworks and science education (bibliography; 3rd ed.). Kiel: IPN.

POSNER, G.J., STRIKE, K.A., HEWSON, P.W. y GERTZOG; W.A. (1982). Accommodation of a scientific conception: toward a theory of conceptual change. Science Education, 66, pp. 211-227.

SCHULMAN, I.S. (1987). Knowledge and teaching: foundations of the new reforms. Harvard Educational Review, 57, pp. 1-22.

SKINNER, B.F. (1953). Science and Hunan Behavior. Nueva York: McMillan Comp.

VAN DRIEL, J.H., DE VOS, W. y VERDONK, A.H. (1990). Why do some molecules react, while others don't?, en Lijnse, P. L. ct al. (eđs.), Relating Macroscopic Phenomena to Microscopic Particles. Utrecht: CDB Press.

VAN KEULEN, H. (1995). Making Sense, Simulation-of-Reseurch in Organic Chemistry. Utrecht: CDB Press.

VERDONK, A.H. y LIJNSE, P.L. (1993). Research in science education at Utrecht University: an outline, en L.jinse, P.L. (ed.), European Research in Science Education, pp. 140-145. Utrecht: CDB Press. 